VOL. $9(1973), 83-88$.

\title{
Invariant metrics on free topological groups
}

\section{Sidney A. Morris and H.B. Thompson}

\begin{abstract}
For a completely regular space $X, G(X)$ denotes the free topological group on $X$ in the sense of Graev. Graev proves the existence of $G(X)$ by showing that every pseudo-metric on $X$ can be extended to a two-sided invariant pseudo-metric on the abstract group $G(X)$. It is natural to ask if the topology given by these two-sided invariant pseudo-metrics on $G(X)$ is precisely the free topological group topology on $G(X)$. If $X$ has the discrete topology the answer is clearly in the affirmative. It is shown here that if $X$ is not totally disconnected then the answer is always in the negative.
\end{abstract}

\section{Introduction}

In [8] and [2] Markov and Graev introduced their respective concepts of free topological group. Graev's concept is more general in the sense that every Markov free topological group is a Graev free topological group. Graev showed that for every completely regular space $X$ his free topological group $G(X)$ always exists. His method of proof was to show that every pseudo-metric on $X$ can be extended to a two-sided invariant pseudo-metric on the abstract group $G(X)$. These pseudo-metrics give rise to a locally invariant topology on the abstract group $G(X)$. It is natural to ask if $G(X)$ with its free topological group topology is locally invariant? This question is of interest since Joiner [4] and Abels [1] have recently investigated the topology on $G(X)$ using pseudo-metrics and related concepts.

Received 9 Harch 1973. 
We show that if $X$ is not totally disconnected then $G(X)$ is not locally invariant. (We note that some restriction on $X$ is necessary since if $X$ is discrete then $G(X)$ is discrete and hence locally invariant.) This result is also of interest as $G(X)$ is always maximally almost periodic [5] and the conditions "maximally almost periodic" and "locally invariant" are "close". For example, for connected locally compact groups the conditions coincide [3].

\section{Notation and preliminaries}

All topological spaces considered will be assumed to be completely regular and Hausdorff. We assume familiarity with the notions of free topo-logical group due to Markov [8] and Graev [2]. Given a topological space $X$, we denote the Graev (Markov) free topological group on $X$ by $G(X) \quad(E(X))$.

We will denote the identity element of a group by $e$. The set of all words in $G(X)$ of length $\leq n$ with respect to $X$ will be denoted by $G_{n}(X)$. We note that $G_{n}(X)=\left(X u X^{-1}\right)^{n}$, for each $n$. Thus if $X$ is compact then each $G_{n}(X)$ is compact.

We will need the following basic structure theorem of Graev [2]. (For an alternative proof of a more general result, see [7].)

THEOREM A. Let $X$ be a compact space. Then a subset $A$ of $G(X)$ is closed if and only if $A \cap G_{n}(X)$ is compact for all $n$.

DEFINITION. A topological group $G$ is said to be locally invariant if every neighbourhood of $e$ contains an invariant neighbourhood of $e$; that is, a neighbourhood of $e$ invariant under all inner automorphisms of $G$.

Note that a topological group is iocally invariant if and only if its topology is given by a family of two-sided invariant pseudo-metrics.

We will use $[0,1]$ to denote the unit interval of the reals with its usual compact topology. The closure of a set $X$ in a topological space will be denoted by cl.X. 


\section{Results}

LEMMA. Let $X$ be any non-totally disconnected space. Then there exists $x \in X$ and closed subsets $A_{n}, n=1,2, \ldots$, of $X$ such that

(i) $x \notin A_{n}$, for alz $n$,

(ii) for any neighbourhood $V$ of $x$ there exists $n$ such that $V \cap A_{n} \neq \emptyset$.

Proof. Since $X$ is not totally-disconnected there exists an infinite closed connected subset $Y$ of $X$.

Suppose that for each $y \in Y$ and every continuous function $f: Y \rightarrow[0,1]$, there exists a neighbourhood $V$ of $y$ such that $f(V)=f(y)$. Since $Y$ is completely regular there exists a function $f: Y \rightarrow[0,1]$ such that $f(Y)$ contains at least two points. Clearly for any $p \in f(Y), f^{-1}(p)$ is an open and closed proper subset of $y$. This is a contradiction.

Thus there exists $y \in Y$ and a continuous function $f: Y \rightarrow[0,1]$ such that for any neighbourhood $V$ of $Y, f(V) \neq f(y)$. Let $S_{n}$ be a countable base of open neighbourhoods at $f(y)$. Put $U_{n}=f^{-1}\left(s_{n}\right)$ and $A_{n}=Y-U_{n}$.

We note that each $A_{n}$ is a closed subset of $X$, and $y \equiv A_{n}$ for any $n$. If $U$ is any neighbourhood in $X$ of $y$, then $U \cap Y$ is a neighbourhood in $Y$ of $y$. Thus $f(U \cap Y) \neq f(y)$. So there exists $v \in U \cap Y$ such that $f(v) \notin S_{n}$ for some $n$. Hence $(U \cap Y) \cap A_{n} \neq \emptyset$. Thus $U \cap A_{n} \neq \emptyset$ for some $n$.

THEOREM. If $X$ is a non-totally disconnected space then $G(X)$ is not locally invariant.

Proof. By the lemma, there exists $x \in X$ and closed subsets $A_{n}$, $n=1,2, \ldots$, of $X$ such that

(i) $x \notin A_{n}$ for any $n$ and 
(ii) for any neighbourhood $V$ of $x$ there exists an $n$ such that $V \cap A_{n} \neq \varnothing$.

Now consider $G(X)$ where the point $x$ is chosen to be the identity $e$. Let $B(X)$ be the Stone-Čech compactification of $X$ [6]. The embedding $\phi: X \rightarrow B(X)$ can be extended to a continuous one-to-one homomorphism $\Phi: G(X) \rightarrow G(B(X))$. Put $B_{n}=c l \cdot\left(\Phi\left(A_{n}\right)\right)$. Noting that $e$ is not an isolated point of $X$ and $e \notin A_{n}$, for any $n$, we can choose distinct points $x_{n} \in X-A_{n}$ such that $x_{n} \neq e$ for $n=1,2, \ldots$. Let $y_{n}=\Phi\left(x_{n}\right)$. Clearly the $y_{n}$ are distinct points, $y_{n} \neq e$ and $y_{n} \in B(X)-B_{n}$, for $n=1,2, \ldots$.

Put $y_{n}=y_{1}^{-1} \ldots y_{n}^{-1} n^{y} y \cdots y_{1}$. Then

(a) each $Y_{n}$ is compact, since $B_{n}$ is compact,

(b) every word in $y_{n}$ is of length $2 n+1$, and

(c) $\Phi^{-1}\left(y_{n}\right)=x_{1}^{-1} \ldots x_{n}^{-1} A_{n} x_{n} \ldots x_{1}=X_{n}$.

Let $B=\bigcup_{n=1}^{\infty} Y_{n}$ and $A=\bigcup_{n=1}^{\infty} X_{n}$. By condition (b), for each $k$, $B \cap G_{k}(B(X))=\bigcup_{n=1}^{k} Y_{n} \cap G_{k}(B(X))$. Since both $Y_{n}$ and $G_{k}(B(X))$ are compact, $B \cap G_{k}(B(X))$ is compact for each $k$. Thus by the structure Theorem $A, B$ is closed in $G(B(X))$. Noting that $A=\Phi^{-1}(B)$ we have $A$ is closed in $G(X)$.

Define $U=G(X)-A$, Then $U$ is an open neighbourhood of $e$. We show that $U$ contains no invariant neighbourhoods of $e$. Suppose $U \supseteq V$, where $V$ is an invariant neighbourhood of $e$. By condition (ii), $V \cap X \cap A_{n} \neq \emptyset$ for some $n$. Thus there exists $v \in V \cap X \cap A_{n}$. Therefore $x_{1}^{-1} \ldots x_{n}^{-1} v x_{n} \ldots x_{1} \in X_{n} \subseteq A$. Hence $x_{1}^{-1} \ldots x_{n}^{-1} V x_{n} \ldots x_{1} \notin V$. So $V$ is not invariant. 
COROLLARY. If $X$ is not totally disconnected then $F(X)$ is not locally invariant.

We conclude our results with an application to the theory of $k \omega^{-s p a c e}$ groups.

DEFINITION. A topological space $X$ is said to be a $k_{\omega}$-space if $X=\bigcup_{n=1}^{\infty} X_{n}$ where each $X_{n}$ is a compact and a subset $A$ of $X$ is closed if and only if $A \cap X_{n}$ is compact for all $n$.

As a prime example of $k_{\omega}$-spaces we have any compact space and any connected locally compact group. For further information see [7], [9], and [10].

As mentioned in the introduction for connected locally compact groups the concepts "maximally almost periodic" and "locally invariant" coincide. One might also expect this to be the case for topological groups which are $k_{\omega}$-spaces. Our final example shows that this is not true.

EXAMPLE. Let $X$ be any compact space which is not totally disconnected. Then by the Structure Theorem $A, G(X)$ is a $k_{\omega}$-space. (This is indeed the case even if $X$ is only a $k_{\omega}$-space which is not totally disconnected [7].) By [5], $G(X)$ is maximally almost periodic, but by our results it is not locally invariant.

\section{References}

[1] Herbert Abels, "Normen auf freien topologischen Gruppen", Math. 2. 129 (1972), 25-42.

[2] М.Н. Граев [M.І. Graev], "Свободные топологические группн" [Free topological groups], Izv. Akad. Nauk SSSR Ser. Mat. 12 (1948), 279-324; Amer. Math. Soc. Trans 2. 35 (1951); reprinted Amer. Math. Soc. Transl. (1) 8 (1962), 305-364.

[3] Siegfried Grosser and Martin Moskowitz, "Compactness conditions in topological groups", J. reine angew. Math. 246 (1971), 1-40. 
[4] Charles Joiner, "Free topological groups and dimension", (to appear).

[5] Shizuo Kakutani, "Free topological groups and infinite direct product topologicel groups", Proc. Imp. Acad. Tokyo 20 (1944), 595-598.

[6] John L. Kelley, General topology (Van Nostrand, Toronto, New York, London, 1955).

[7] John Mack, Sidney A. Morris, Edward T. Ordman, "Free topological groups and the projective dimension of a locally compact abelian group", Proc. Amer. Nath. Soc. (to appear).

[8] A.A. Mарков [A.A. Markov], "0 свободных топологическнх группах" [On free topological groups], Izv. Akad. Nauk SSSR Ser. Mat. 9 (1945), 3-64; Amer. Math. Soc. Trans 2. 30 (1950), 11-88; reprinted Amer. Math. Soc. Trans 2. (1) 8 (1962), 195-272.

[9] Ernest Michael, "Bi-quotient maps and Cartesian products of quotient maps", Ann. Inst. Fourier (Grenoble) 18 (1968), fasc. 2, 287-302, vii (1969).

[10] Edward T. Ordman, "Free products of topological groups which are $k_{w}$-spaces", Trans. Amer. Math. Soc. (to appear).

School of Mathematics,

University of New South Wales,

Kensington,

New South Wales;

School of Mathematical Sciences,

Flinders University,

Bedford Park,

South Australia. 